“C 2010 IEEE. Personal use of this material is permitted. Permission from IEEE must be obtained for all other uses, in any current or future media, including reprinting/republishing this material for advertising or promotional purposes, creating new collective works, for resale or redistribution to servers or lists, or reuse of any copyrighted component of this work in other works. 


\title{
Torque Ripples and Estimation Performance of High Frequency Signal Injection based Sensorless PMSM Drive Strategies
}

\author{
Yi Wang ${ }^{1}$ \\ Youguang Guo ${ }^{1}$ \\ Senior Member

\begin{abstract}
The developed nonlinear permanent magnet synchronous machine (PMSM) model established a virtual model to simulate and assess the sensorless drive schemes for PMSMs avoiding the experimental trial and error method. In this paper, three sensorless PMSM drive schemes are investigated and compared, which are all based on the high frequency signal injection. The nonlinear PMSM machine model is employed to accurately simulate the machine performance and to analytically assess the efficiency of the sensorless algorithm. Both the structural and saturation saliencies are incorporated in this comprehensive model. The comparison of the machine torque ripples induced by excitation signals are carried out. The magnitude of injected signal and recordable rotor position information are considered together as the efficiency of the estimation algorithm. Additionally, the implementation and signal process computing costs are considered for all the schemes. Finally, a comprehensive comparison of all the methods is developed.
\end{abstract}

\author{
Jianguo $\mathrm{Zhu}^{1}$ \\ Senior Member
}

Yongjian $\mathrm{Li}^{2}$

\author{
Wei Xu ${ }^{1}$ \\ ${ }^{1}$ School of Electrical, Mechanical and Mechatronic Systems, University of Technology, Sydney, Australia \\ Po Box 123 Broadway NSW 2007 Australia \\ ${ }^{2}$ Province-Ministry Joint Key Laboratory of Electromagnetic Field and Electrical Apparatus Reliability, \\ Hebei University of Technology, PO Box 359, No. 8 Guangrong Road, Tianjin 300130, China \\ wang.yi@uts.edu.au
}

Index Terms-nonlinear model, permanent magnet synchronous machine, saturation saliency, sensorless drive, structural saliency, torque ripple

\section{NOMENCLATURE}

Symbols and Abbreviations

\begin{tabular}{|c|c|}
\hline$\lambda_{\alpha}^{*}, \lambda_{\beta}^{*}$ & Projected flux linkages on $\alpha$ - and $\beta$-axis \\
\hline$\lambda_{d}^{*}, \lambda_{q}^{*^{\prime}}$ & Projected flux linkages on $d$ - and $q$-axis \\
\hline$v_{\alpha k}, v_{\beta h}$ & $\begin{array}{l}\text { High frequency component of } \alpha \text { - and } \beta \text {-axis } \\
\text { voltages }\end{array}$ \\
\hline$i_{\alpha h}, i_{\beta h}$ & $\begin{array}{l}\text { High frequency component of } \alpha \text { - and } \beta \text {-axis } \\
\text { currents }\end{array}$ \\
\hline$v_{c}$ & $\begin{array}{l}\text { High frequency component of } d \text { - and } q \text {-axis } \\
\text { voltages }\end{array}$ \\
\hline$h^{h}$ & $\begin{array}{l}\text { High frequency component of } d \text { - and } q \text {-axis } \\
\text { currents }\end{array}$ \\
\hline$D$ & Stator resistance \\
\hline
\end{tabular}

$\begin{array}{ll}K_{\alpha}, K_{\beta} & \begin{array}{l}\text { Structural saliency factors of the } \alpha \text { - and } \beta- \\ \text { axis }\end{array} \\ K_{d}, K_{q} & \begin{array}{l}\text { Structural saliency factors of the } d \text { - and } q \text { - } \\ \text { axis }\end{array} \\ K_{s t r} & \text { StrSR: Structural saliency ratio } \\ K_{\text {sat }} & \text { SatSR: Saturation saliency ratio } \\ & \text { II. INTRODUCTION }\end{array}$

The permanent magnet (PM) electrical machines, e.g. the permanent magnet synchronous machine (PMSM), have found wide applications due to their high power density (compactness), high efficiency, ease of control, high torqueto-inertia ratio, and high reliability. A lot of efforts then have been made to improve the PMSM drive performance from the aspects of power electronics, control theory or computing techniques [1]-[3]. However, the application of PMSMs is limited because of the unavoidable rotor position sensor, which not only increases the system cost, but also perhaps more importantly reduces the system reliability.

In recent years, numerous papers have been published that address the sensorless control methods to eliminate the mechanical position and/or speed sensors, among which the high frequency signal injection technique is widely employed. An external electrical signal is always injected into the stator windings and the rotor position information is observed based on the measured stator currents or voltages. The injected signal is always chosen as high frequency $\mathrm{AC}$ signal or a series of short DC pulses [4] [5] so that the excitation signal will not interrupt the machine normal operation. On the other hand, the rotor position information contained in the high frequency feedback is easier to be abstracted from the fundamental machine electrical signals by applying some signal processing techniques.

Based on the injection direction of the excitation signal, the 
high frequency injection schemes could be classified as rotating injection method, in which the carrier signal is a rotating sinusoidal signal in the stationary reference frame [6] [7]; $d$-axis injection method, in which an AC voltage signal is injected on the estimated rotor $d$-axis, rotating with the rotor [8] and the rotor position offset could be observed from the high frequency component of the estimated $q$-axis current. However, the drive efficiency of the schemes is hard to be accessed and the development of the sensorless strategies suffers the unknown nonlinearity of the model.

The traditional PMSM model is based on the linear magnetic property assumption that the inductance of the stator winding is a constant value for different stator currents. The linear model functions reasonably well in describing the energy conversion and the torque generation inside a PMSM. The widely used field oriented control (FOC) and direct torque control (DTC) methods were developed based on this linear model and achieved good control performance.

However, there is not an analytical solution for initial rotor position detection and sensorless drive method developed because of the lack of accurate nonlinear machine model incorporating the magnetic saliencies. The linear model is not sufficient to accurately simulate the machine performance and describe the machine magnetic nonlinearities. Experimental trial and error method is commonly employed to develop and verify the sensorless drive schemes. At the same time, there is not a uniform method to access the sensorless drive methods yet. On the other hand, the machine state observers developed based on simplified mathematic model would lead to errors on the control side.

Several modeling methods have been proposed to incorporate the magnetic nonlinearity. Considerations were taken to calculate the machine inductance variation associated with the saturation effect [9] and the mutualinductance between the orthogonal axes [10]. However, those models are always established based on finite element method (FEM), which requires huge computing cost and is hard to be applied for dynamic performance simulation [11].

In [12], the nonlinear saturation effect, as well as the structural saliency, is incorporated and expressed in the new analytical machine model. Two factors, the structural saliency ratio (StrSR) and the saturation saliency ratio (SatSR), are defined to indicate the extent of magnetic saliencies. The mathematic model of PMSM is derived in both the stator and rotor reference frames. The nonlinear inductance matrix is expressed as a function of the stator current and the rotor position.

Based on this nonlinear PMSM model, the $\alpha$-axis injection method is proposed in this paper, in which an $\mathrm{AC}$ voltage signal is always injected on stator $\alpha$-axis and the rotor position information is observed from the high frequency component of the stationary $\beta$-axis current. Then the rotor position estimation methods are investigated and compared. The performance comparison is conducted based on the torque ripple, drive efficiency and cost. An overall performance comparison is then carried out.

\section{NONLINEAR PMSM MODEL}

The magnetic saliencies in a PMSM could be classified as the structural saliency that mainly comes from the salient structure, and the saturation saliency induced by the magnetic saturation inside the core [13]. For a given PMSM, these saliencies could be decoupled since the saturation is due to the nonlinear magnetic property of the core while the structural saliency is not related to the magnetic nonlinearity.

\section{A. Saliency Ratios: StrSR and SatSR}

In a PMSM, the total flux linking the stator windings can be attributed to two sources - the rotor permanent magnets and the stator $\mathrm{AC}$ currents. Because of the nonlinear saturation effect, the total flux cannot be separated into flux spatial vectors due to the permanent magnet and stator current, respectively. In order to express the extent of magnetic saliencies, two parameters, StrSR and SatSR, are defined for the structural and saturation saliencies, respectively.

The structural saliency is always prefixed for a PMSM when it has been designed. It is formed by the structure of the rotor magnet and usually easy to obtain from the unbalanced $d$ - and $q$-axis inductances in the machine model.

To express and analyze the structural saliency, the average linear inductance and StrSR are respectively defined as

$$
\begin{aligned}
& L_{\alpha v}=\frac{L_{d}+L_{q}}{2} \\
& K_{s t r}=\frac{L_{d}-L_{q}}{L_{d}+L_{q}}
\end{aligned}
$$

where $L_{d}$ and $L_{q}$ are respectively the $d$ - and $q$-axis inductance without excitation in the stator.

In the traditional SPMSM model, StrSR is usually assumed to be zero because the structural saliency is very small. In this paper, it is considered and expressed.

The saturation saliency is caused by the saturation effect of the stator core and exists in both interior PMSMs (IPMSMs) and SPMSMs. It is usually discussed for SPMSM because it is the main saliency inside an SPMSM, where the structural saliency is always ignorable.

As discussed above, the saturation of the stator core mainly takes place in the direction of the total flux vector. As a result, the inductance in this direction can be treated as a function of the equivalent total current, not a constant as defined in the traditional PMSM model. Based on the saturation curve, SatSR is then defined as 


$$
K_{s a t}\left(i_{0}\right)=\frac{\frac{\lambda\left(i_{0}\right)}{i_{0}}-\left.\frac{d \lambda}{d i}\right|_{i=i_{0}}}{\frac{\lambda\left(i_{0}\right)}{i_{0}}}
$$

As shown in (3), SatSR is also a function of the current and the linear part will be discussed later after modeling

\section{B. Nonlinear Model of PMSM}

In order to compare easily with the rotor $d-q$ reference frame, the two-phase orthogonal $\alpha-\beta$ stationary reference frame is used to express the machine electrical model instead of $a-b-c$ frame. Equivalent current vectors, $i_{t}^{*}$ and $i_{f}^{*}$ are defined, which produces the total flux linkage and the rotor flux linkage. As shown in Fig. $1, i_{t}^{*}$ is not equal to $i_{t}$, which is a combination of the stator and rotor currents, because of the structural and saturation saliencies.

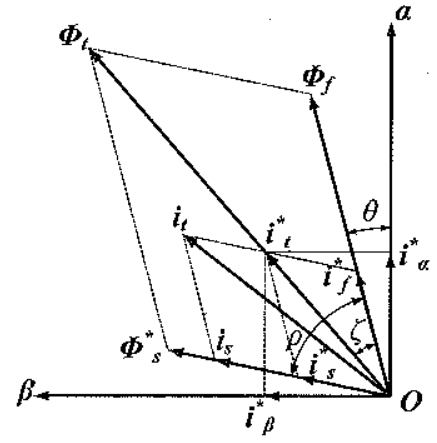

Fig. 1. Phasor diagram in $\alpha-\beta$ stationary reference frame

The flux in the air-gap can be treated as a sinusoidal distribution for most PMSMs. Therefore the flux linkages on the $\alpha$ - and $\beta$-axis are defined as the projection from the total flux linkage or called the equivalent flux linkage. The voltage equations of PMSM machine can be described as

$$
\left\{\begin{array}{l}
v_{\alpha}=R i_{\alpha}+\frac{d}{d t} \lambda_{\alpha}^{*} \\
v_{\beta}=R i_{\beta}+\frac{d}{d t} \lambda_{\beta}^{*}
\end{array}\right.
$$

where $v_{\alpha}, v_{\beta}, i_{\alpha}$ and $i_{\beta}$ are the stator voltage and current in the $\alpha-\beta$ stationary reference frame, $R$ is the stator resistance, and $\lambda_{\alpha}^{*}, \lambda_{\beta}^{*}$ are the projected flux linkages on the $\alpha$ - and $\beta$-axes.

The structural saliency factors of the $\alpha$ - and $\beta$-axis are defined as $K_{\alpha}$, and $K_{\beta}$, with the assumption that the linear inductance follows a spatially sinusoidal distribution, and they are expressed as

$$
\left\{\begin{array}{l}
K_{\alpha}=\frac{1+K_{s t r} \cos 2 \theta}{1+K_{s t r} \cos 2 \rho} \\
K_{\beta}=\frac{1-K_{s t r} \cos 2 \theta}{1+K_{s t r} \cos 2 \rho}
\end{array}\right.
$$

Additionally, $d \lambda_{t}^{*} / d i_{t}^{*}$ and $\lambda_{t}^{*} / i_{t}^{*}$ are not equal in the nonlinear machine model due to the saturation effect. The linear inductance $\lambda_{t}^{*} / i_{t}^{*}$ is then defined as $L_{t}$ and expressed as

$$
\left\{\begin{array}{l}
\frac{\lambda_{t}^{*}}{i_{t}^{*}}=L_{t} \\
\frac{d \lambda_{t}^{*}}{d i_{t}^{*}}=\left(1-K_{s a t}\right) L_{t}
\end{array}\right.
$$

Finally, the machine voltage equation (4) can be rewritten

$$
\left[\begin{array}{c}
v_{\alpha} \\
v_{\beta}
\end{array}\right]=R\left[\begin{array}{l}
i_{\alpha} \\
i_{\beta}
\end{array}\right]+\left[\begin{array}{ll}
L_{\alpha \alpha} & L_{\alpha \beta} \\
L_{\beta \alpha} & L_{\beta \beta}
\end{array}\right] \frac{d}{d t}\left[\begin{array}{l}
i_{\alpha} \\
i_{\beta}
\end{array}\right]+\left[\begin{array}{c}
e_{\alpha} \\
e_{\beta}
\end{array}\right]
$$

where $e_{\alpha}$ and $e_{\beta}$ are the back-emf and the nonlinear inductance matrix can be expressed as

$$
\begin{aligned}
& {\left[\begin{array}{ll}
L_{\alpha \alpha x} & L_{\alpha \beta} \\
L_{\beta \alpha} & L_{\beta \beta}
\end{array}\right]} \\
& =\left[\begin{array}{cc}
L_{t} K_{\alpha}\left(1-K_{\text {sat }} \cos ^{2}(\zeta+\theta)\right) & -\frac{1}{2} L_{t} K_{\beta} K_{s a t} \sin 2(\zeta+\theta) \\
-\frac{1}{2} L_{t} K_{\alpha} K_{\text {sat }} \sin 2(\zeta+\theta) & L_{t} K_{\beta}\left(1-K_{s a t} \sin ^{2}(\zeta+\theta)\right)
\end{array}\right]
\end{aligned}
$$

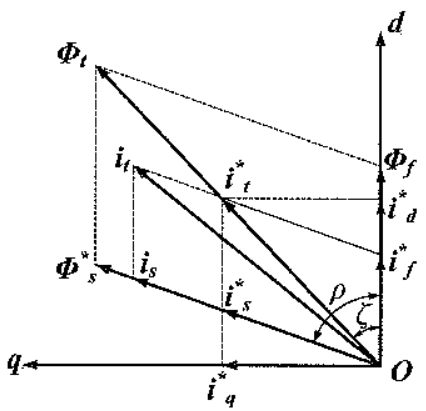

Fig. 2. Phasor diagram in $d-q$ rotor reference frame

On the other hand, rotating orthogonal $d-q$ axis is a widely used rotor field oriented reference frame, where the stator side variables could be converted into rotor side rotating with the rotor field. The projected current and voltage vectors are relatively motionless to the rotor flux. The Park-Clark transform can also be applied to the nonlinear machine model because the flux vectors are all obtained from the total flux 
linkage projection. Then the voltage equation

$$
\left[\begin{array}{l}
v_{d} \\
v_{q}
\end{array}\right]=R\left[\begin{array}{l}
i_{d} \\
i_{q}
\end{array}\right]+\frac{d}{d t}\left[\begin{array}{l}
\lambda_{d}^{*} \\
\lambda_{q}^{*}
\end{array}\right]+\left[\begin{array}{c}
-\lambda_{q}^{*} \\
\lambda_{d}^{*}
\end{array}\right] \omega_{e}
$$

is valid for nonlinear modeling, where $\lambda_{d}^{*}$ and $\lambda^{*}$ are the equivalent flux linkages on the $d$ - and $q$-axes which are projected from the total flux linkage. The phasor diagram in the $d-q$ reference frame is shown in Fig. 2.

The flux linkages in (9) can be expressed by the total flux linkage and the angle as below

$$
\left\{\begin{array}{l}
\lambda_{d}^{*}=\lambda_{t}^{*} \cos \zeta \\
\lambda_{q}^{*}=\lambda_{t}^{*} \sin \zeta
\end{array}\right.
$$

Similar as in stationary reference frame, the structural saliency factors of the $d$ - and $q$-axes are defined as

$$
\left\{\begin{array}{l}
K_{d}=\frac{1+K_{s t r}}{1+K_{s t r} \cos 2 \rho} \\
K_{q}=\frac{1-K_{s t r}}{1+K_{s t r} \cos 2 \rho}
\end{array}\right.
$$

Based on the same definition of the linear inductance as in (6), the PMSM voltage equation in the rotor reference frame can be rewritten as

$$
\left[\begin{array}{l}
v_{d} \\
v_{q}
\end{array}\right]=R\left[\begin{array}{l}
i_{d} \\
i_{q}
\end{array}\right]+\left[\begin{array}{ll}
L_{d d} & L_{d q} \\
L_{q d} & L_{q q}
\end{array}\right] \frac{d}{d t}\left[\begin{array}{l}
i_{d} \\
i_{q}
\end{array}\right]+\left[\begin{array}{c}
-\lambda_{q}^{*} \\
\lambda_{d}^{*}
\end{array}\right] \omega_{e}
$$

where the nonlinear inductance matrix can be expressed as

$$
\left[\begin{array}{ll}
L_{d d} & L_{d q} \\
L_{q d} & L_{q q}
\end{array}\right]=\left[\begin{array}{cc}
L_{t} K_{d}\left(1-K_{s a t} \cos ^{2} \zeta\right) & -\frac{1}{2} L_{t} K_{q} K_{s a t} \sin 2 \zeta \\
-\frac{1}{2} L_{t} K_{d} K_{s a t} \sin 2 \zeta & L_{t} K_{q}\left(1-K_{s a t} \sin ^{2} \zeta\right)
\end{array}\right]
$$

From (12) and (13), the traditional PMSM linear model can be obtained as an ideal condition, where the saturation saliency is ignored, $K_{\text {sat }}=0$, as shown below

$$
\left[\begin{array}{l}
v_{d} \\
v_{q}
\end{array}\right]=R\left[\begin{array}{c}
i_{d} \\
i_{q}
\end{array}\right]+\left[\begin{array}{cc}
L_{d} & 0 \\
0 & L_{q}
\end{array}\right] \frac{d}{d t}\left[\begin{array}{l}
i_{d} \\
i_{q}
\end{array}\right]+\left[\begin{array}{c}
-\lambda_{q} \\
\lambda_{d}
\end{array}\right] \omega_{e}
$$

\section{Linear Inductance Component}

The linear inductance component defined in (6) is still a function of the stator current and the rotor position. Based on the magnetization curve, the linear inductance $\lambda_{t}^{*} / i_{t}^{*}$ variation is always smaller than that of the differential inductance $d \lambda_{y}^{*} / d i_{t}^{*}$ and could be neglected. Then the linear inductance component is assumed to be fixed and expressed by using the given $L_{d}$ and $L_{q}$ values, which are always known for the machine. The machine inductance matrixes in (8) and (13) could be rewritten as

$$
\begin{aligned}
& {\left[\begin{array}{ll}
L_{\alpha \alpha x} & L_{\alpha \beta} \\
L_{\beta \alpha} & L_{p \beta \beta}
\end{array}\right]=\left[\begin{array}{l}
L_{a v}\left(1+K_{s t r} \cos 2 \theta\right)\left(1-K_{s a t} \cos ^{2}(\zeta+\theta)\right) \\
-\frac{1}{2} L_{a v}\left(1+K_{s t r} \cos 2 \theta\right) K_{s a t} \sin 2(\zeta+\theta)
\end{array}\right.} \\
& \left.\begin{array}{r}
-\frac{1}{2} L_{a v}\left(1-K_{s t r} \cos 2 \theta\right) K_{s a t} \sin 2(\zeta+\theta) \\
L_{a v}\left(I-K_{s t r} \cos 2 \theta\right)\left(1-K_{s a t} \sin ^{2}(\zeta+\theta)\right)
\end{array}\right]
\end{aligned}
$$

where $L_{a v}=\left(L_{d}+L_{q}\right) / 2$ is the average linear inductance in the $d$ and $q$-axes expressed as

$$
\left[\begin{array}{ll}
L_{d d} & L_{d q} \\
L_{q d} & L_{q q}
\end{array}\right]=\left[\begin{array}{cc}
L_{d}\left(1-K_{s a t} \cos ^{2} \zeta\right) & -\frac{1}{2} L_{q} K_{s a t} \sin 2 \zeta \\
-\frac{1}{2} L_{d} K_{s a t} \sin 2 \zeta & L_{q}\left(1-K_{s a t} \sin ^{2} \zeta\right)
\end{array}\right]
$$

\section{VIRTUAL MACHINE MODEL}

In order to build up a virtual machine model, an SPMSM machine is chosen as the prototype. The machine parameters are shown as in Table I.

TABLE I

PARAMETERS OF TESTED SPMSM

\begin{tabular}{cc}
\hline Parameter & Value \\
\hline Model & IFT 6071-6AC21-2-Z \\
Number of Poles & 6 \\
Rated Power & $1000 \mathrm{~W}$ \\
Rated Voltage & $128 \mathrm{~V}$ \\
Rated Current & $6.5 \mathrm{~A}$ \\
Rated Speed & $2000 \mathrm{rev} / \mathrm{min}$ \\
Rated Torque & $4.8 \mathrm{Nm}$ \\
\hline
\end{tabular}

The tested SPMSM has a small structural saliency, but a reasonably large saturation saliency. The inductance values and structural saliency are identified as

$$
\left\{\begin{array}{l}
L_{d}=0.0142 H \\
L_{q}=0.0159 H \\
L_{a v}=0.0151 H \\
K_{s t r}=-5.65 \%
\end{array}\right.
$$

On the other hand, SatSR always depends on the nonlinear magnetization curve of the material and cannot be analytically expressed. The $d$-axis inductance is a function of SatSR for different current levels. Several tests have been carried out to check the SatSR as in [12]. The relationship 
between the current magnitude and the SatSR is shown in Table II.

TABLE II

RELATIONSHIP BETWEEN CURRENT AND SATSR

\begin{tabular}{ccc}
\multicolumn{3}{c}{$\begin{array}{c}\text { RELATIONSHP BETWEEN CURRENT AND SATSR } \\
\left(\lambda_{f}=0.1495 \mathrm{~Wb}, \zeta=0, K_{\text {str }}=5.65 \%\right)\end{array}$} \\
\hline $\begin{array}{ccc}\text { Current Offset } \\
i_{s}(\mathrm{~A})\end{array}$ & $\begin{array}{c}\text { Average d-inductance } \\
L_{d d}(\mathrm{mH})\end{array}$ & $\begin{array}{c}\text { SatSR } \\
K_{\text {sat }}(\%)\end{array}$ \\
\hline 0 & 14.23 & 0 \\
1 & 14.14 & 0.60 \\
2 & 14.08 & 1.02 \\
3 & 13.91 & 2.23 \\
4 & 13.74 & 3.39 \\
5 & 13.57 & 4.59 \\
6 & 13.33 & 6.33 \\
\hline
\end{tabular}

Based on this virtual model, the mutual-inductances between the orthogonal axes are calculated at different current offsets. Fig. 3 shows the mutual-inductance $L_{\alpha \beta}$ under different stator currents, where a sinusoidal curve $K \cdot \sin (2 \theta)$ is added to indicate the rotor position.

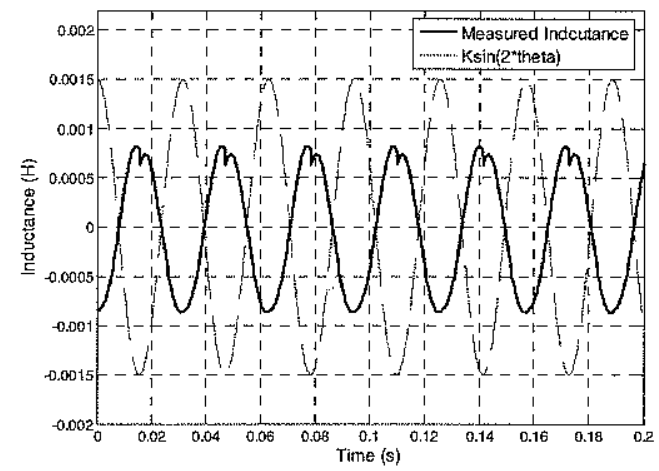

(a). $L_{a \beta}$, stator current $=1$ A.

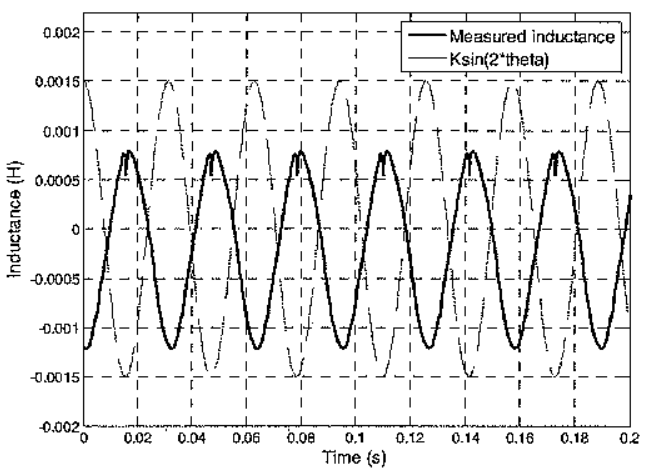

(b). $L_{\alpha \beta,}$ stator current $=5 \mathrm{~A}$.

Fig. 3. The mutual-inductance between $\alpha$-and $\beta$-axes at different stator current.

\section{ROTOR POSITION DETECTION METHODS}

The analytical machine model enables the accurate performance simulation and efficiency comparison of different sensorless strategies. Furthermore, new injection method could also be proposed based on the analytical expression as well.

\section{A. Rotating Carrier Injection Method}

In the rotating carrier signal injection method, a high frequency rotating voltage vector is superimposed on the fundamental excitation and analysis of the resulting high frequency current is conducted to track spatial saliencies of the magnetic field. The injected rotating voltage can be expressed as (18).

$$
\left[\begin{array}{l}
v_{\alpha h} \\
v_{\beta h}
\end{array}\right]=\left[\begin{array}{l}
V_{i n} \cos \omega_{h} t \\
V_{i n} \sin \omega_{h} t
\end{array}\right]
$$

where $\omega_{h}$ is the injected signal frequency; $V_{i n}$ is the injected voltage magnitude.

If the high frequency component of voltages and currents are only considered and the rotor speed of the machine is relatively small compared to the injected signal frequency, the machine voltage equation, (4) could be rewritten as

$$
\left[\begin{array}{c}
i_{\alpha h} \\
i_{\beta h}
\end{array}\right]=\left[\begin{array}{ll}
Z_{\alpha \alpha \alpha} & Z_{\alpha \beta} \\
Z_{\beta \alpha} & Z_{\beta \beta}
\end{array}\right]^{-1}\left[\begin{array}{l}
v_{\alpha b h} \\
v_{\beta h}
\end{array}\right]
$$

For steady state operation, the StrSR and SatSR are fixed and relatively small compared to one. Then the expression could be simplified. The high frequency current component could be derived as

$v_{o d}=K_{h}\left\{\frac{1}{2} \sin 2 \theta \sin \omega_{h} t\left[L_{q}\left(1-K_{s a t} \sin ^{2} \zeta\right)-L_{d}\left(1-K_{s a t} \cos ^{2} \zeta\right)\right]\right.$ $+\frac{1}{2} K_{\text {sat }} \sin 2 \zeta\left[\sin \omega_{h} t\left(L_{d} \cos ^{2} \theta-L_{q} \sin ^{2} \theta\right)-L_{\alpha v} \sin 2 \theta \cos \omega_{h} t\right]$ $\left.+\cos \omega_{h} t\left[L_{q}\left(1-K_{s a t} \sin ^{2} \zeta\right) \cos ^{2} \theta+L_{d}\left(1-K_{s a t} \cos ^{2} \zeta\right) \sin ^{2} \theta\right]\right\}$

$v_{\beta h}=K_{h}\left\{\frac{1}{2} \sin 2 \theta \cos \omega_{h} t\left[L_{q}\left(1-K_{s a t} \sin ^{2} \zeta\right)-L_{d}\left(1-K_{s a t} \cos ^{2} \zeta\right)\right]\right.$ $+\frac{1}{2} K_{s a t} \sin 2 \zeta\left[L_{\alpha}, \sin 2 \theta \sin \omega_{h} t-\cos \omega_{h} t\left(L_{d} \sin ^{2} \theta-L_{q} \cos ^{2} \theta\right)\right]$ $\left.+\sin \omega_{h} t\left[L_{q}\left(1-K_{s a t} \sin ^{2} \zeta\right) \sin ^{2} \theta+L_{d}\left(1-K_{s a t} \cos ^{2} \zeta\right) \cos ^{2} \theta\right]\right\}$

where $K_{h}=\frac{V_{\text {in }}}{j \omega_{h} L_{d} L_{q}\left(1-K_{s a t}\right)}$.

As in (20) and (21), the rotating component of the carrierfrequency current contains the desired information about the inductance saliency in its phase. It is noted that the second part of the current expression depends on the saturation saliency and the third part is almost zero after demodulation and signal processing. This estimation method is mainly based on the rotor structural saliency, which is indicated in 
the first part.

In order to estimate the rotor position from the phase current vector, a tracking observer is added to extract the rotor position with filtering of unwanted noise. The output of the signal processing unit or the effective magnitude of rotor position information could be simplified as

$$
v_{o} \approx \frac{1}{2} K_{h} \sin 2 \theta\left[L_{q}\left(1-K_{s a t} \sin ^{2} \zeta\right)-L_{d}\left(1-K_{s a t} \cos ^{2} \zeta\right)\right]
$$

\section{B. d-axis Injection Method}

This method is also named as oscillating vector injection or AC pulse injection method, where a $d$-axis sinusoidal carrier signal in the estimated rotor reference frame is superimposed onto the fundamental component voltage vector.

The estimated rotor reference frame is established after the initial rotor position is identified and rotates with the machine. The injected signal frequency is sufficiently high compared with the estimated rotor speed, the voltage and current in the estimated rotor reference frame can be expressed as

$$
\left[\begin{array}{c}
\hat{v_{d h}} \\
\hat{v_{q h}}
\end{array}\right]=\left[\begin{array}{c}
V_{i n} \cos \omega_{h} t \\
0
\end{array}\right]
$$

Therefore, the voltage vector transferred to the rotating reference frame yields

$$
\left[\begin{array}{c}
v_{d h} \\
v_{q h}
\end{array}\right]=V_{i n} \cos \omega_{h} t\left[\begin{array}{c}
\cos \Delta \theta \\
-\sin \Delta \theta
\end{array}\right]
$$

where $\Delta \theta$ is the error angle of the estimated $d$-axis.

The components of the high frequency current in the estimated rotor position reference frame is given by

$$
\begin{aligned}
\hat{v_{d h}} & =K_{h} \cos \omega_{h} t\left[\frac{1}{2} K_{s a t} L_{a v} \sin 2 \zeta \sin 2 \Delta \theta\right. \\
& \left.+L_{d}\left(1-K_{s a t} \cos ^{2} \zeta\right) \sin ^{2} \Delta \theta-L_{q}\left(1-K_{s a t} \sin ^{2} \zeta\right) \cos ^{2} \Delta \theta\right]
\end{aligned}
$$

$$
\begin{aligned}
\hat{v_{q h}} & =\frac{1}{2} K_{h} \cos \omega_{h} t\left\{K_{s a t} \sin 2 \zeta\left(L_{q} \cos ^{2} \Delta \theta-L_{d} \sin ^{2} \Delta \theta\right)\right. \\
& \left.+\sin 2 \Delta \theta\left[L_{q}\left(1-K_{s a t} \sin ^{2} \zeta\right)-L_{d}\left(1-K_{s a t} \cos ^{2} \zeta\right)\right]\right\}
\end{aligned}
$$

The carrier-frequency current for this oscillating vector injection in the estimated rotor reference frame has the desired inductance saliency information in its amplitude, while the same information is in the phase of the carrierfrequency current for the rotating vector injection in the stationary reference frame. The $q$-axis component at the carrier frequency is used to obtain the position error. The first part of the $q$-axis current represents the saturation saliency and it is relatively small than the second part, which indicates the structural saliency based rotor axis position. The output of the signal processing unit or the effective magnitude of rotor position error could be simplified as

$v_{o} \approx \frac{1}{2} K_{h} \sin 2 \Delta \theta\left[L_{q}\left(1-K_{s a t} \sin ^{2} \zeta\right)-L_{d}\left(1-K_{s a t} \cos ^{2} \zeta\right)\right]$

\section{C. $\alpha$-axis Injection Method}

As shown in Fig. 3, the mutual-inductance between $\alpha$ - and $\beta$-axes, $L_{\alpha \beta}$ is a second order function of rotor electrical position. Therefore, it is possible to estimate the rotor position from $\beta$-axis current response when an $\mathrm{AC}$ signal is set up to be injected into $\alpha$-axis.

A simplified position sensorless scheme is then proposed as $\alpha$-axis injection, in which the AC pulse or carrier signal is injected on the stationary $\alpha$-axis as (28).

$$
\left[\begin{array}{l}
v_{\alpha h} \\
v_{\beta h}
\end{array}\right]=\left[\begin{array}{c}
V_{i n} \cos \omega_{h} t \\
0
\end{array}\right]
$$

The high frequency current component on $\beta$-axis could be derived as

$$
\begin{aligned}
& v_{o h h}=\frac{V_{i n} \cos \omega_{h} t}{j 2 \omega_{h}} L_{\alpha v}\left(1-K_{s t r} \cos 2 \theta\right)\left(1-K_{s a t} \sin ^{2}(\zeta+\theta)\right) \\
& v_{\beta h}=\frac{V_{i n} \cos \omega_{h} t}{j 2 \omega_{h}} L_{a v}\left(1-K_{s t r} \cos 2 \theta\right) K_{s a t} \sin 2(\zeta+\theta)
\end{aligned}
$$

It could be found that the rotor position $I$ information could be abstracted from the $\beta$-axis current component and this rotor position depends on the saturation saliency. The output of the signal processing unit or the effective magnitude of rotor position error could be simplified as

$$
v_{o} \approx \frac{V_{i n}}{j 2 \omega_{h}} L_{\alpha v} K_{s a t} \sin 2(\zeta+\theta)
$$

\section{PERformance Comparison}

In MATLAB/SIMULINK, a virtual machine model is established and the sensorless strategies above are simulated. In rotating carrier signal injection scheme, there is not reference frame transformation required for the excitation signal. The three-phase high frequency signal is generated and applied to the stator with the fundamental voltage signals. The implementation cost is very small. Signal process part is needed to abstract the negative-sequence high frequency current component. The collected information is a function of the injected voltage magnitude, frequency and the machine 
structural saliency. As an external signal injection, the torque ripples caused by the high frequency signal is unavoidable. Fig. 4 shows the electromagnetic torque output of the machine under the rotating carrier signal injection. Vector control is applied to drive the machine.

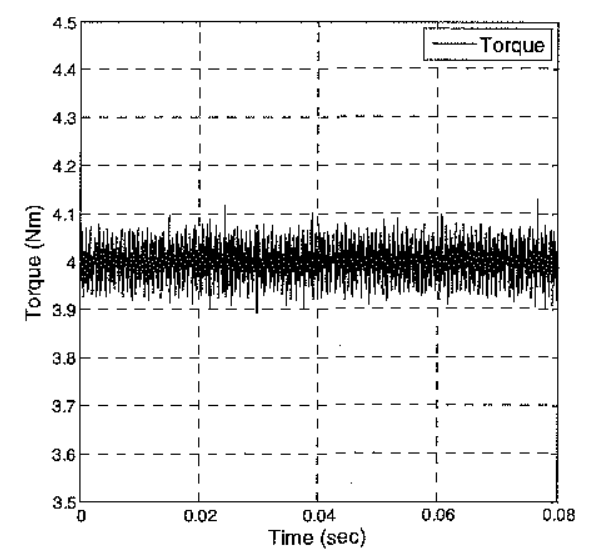

Fig. 4. Torque output of rotating carrier injection method. $\left(\omega^{m=100 \pi} \mathrm{rad} / \mathrm{s}\right.$, $f_{h}=500 \mathrm{~Hz}, T_{L}=4 \mathrm{Nm}$ )

Secondly, in the $d$-axis injection scheme, the injection reference frame is rotating with the rotor. Reference frame transformation is needed to carry out the injection signal in stationary frame. For the rotor position observer, the signal process part has simple structure, in which only $q$-axis current component at carrier-frequency is needed. On the other hand, the rotor position error is smaller because the position tracking is designed to minimize the angle error between the real and estimation reference frames. For a perfect $d$-axis injection sensorless drive, the external signal only takes effect on $d$-axis and there is not additional torque ripples generated. However, there is always an estimation error in the drive system and the torque ripples are not longer zero. Fig. 5 shows the electromagnetic torque output when the rotor angle estimation error is $\pm 0.2 \mathrm{rad}$.

For $\alpha$-axis injection method, the excitation AC signal has fixed direction in stationary frame, which can be generated by using a simple implementation procedure. Similar as the signal process in $d$-axis injection method, only $\beta$-axis current component at carrier-frequency is needed to estimate the rotor position. On the other hand, this method is mainly based on the nonlinearity and saturation saliency of the machine. Therefore, it can be applied to both IPMSM and SPMSM. However, some unexpected torque ripples will be generated, especially when the rotor $q$-axis rotates to the position near the excited $\alpha$-axis. Fig. 6 shows the torque ripples of $\alpha$-axis injection method. The periodic torque ripples could be identified in the current feedback loop. Therefore, this ripple could be partially eliminated by modifying the PI controller parameters or applying other advance current regulators.

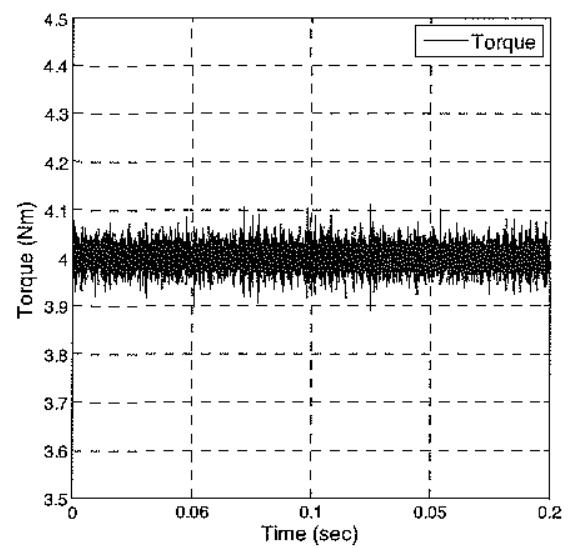

Fig. 5. Torque output of $d$-axis injection method. $\Delta \theta= \pm 0.2 \mathrm{rad}$. ( $\omega=100 \pi$ $\mathrm{rad} / \mathrm{s}, f_{h}=500 \mathrm{~Hz}, T_{L}=4 \mathrm{Nm}$ )

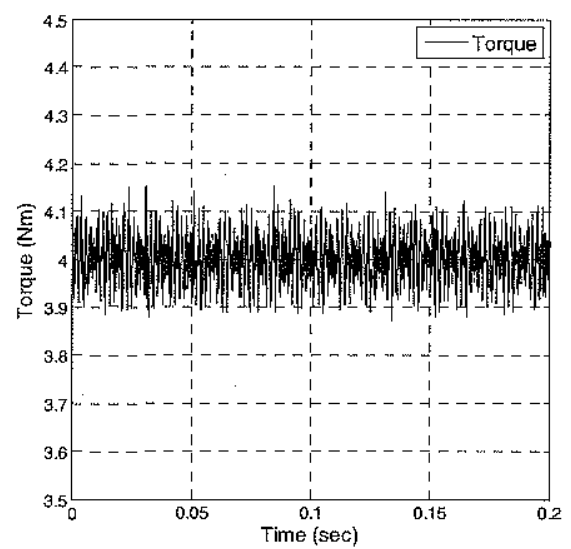

Fig. 6. Torque output of $\alpha$-axis injection method. ( $\omega=100 \pi \mathrm{rad} / \mathrm{s}, f_{h}=500$ $\mathrm{Hz}, T_{l}=4 \mathrm{Nm}$ )

As shown above, the $d$-axis injection method could achieve minimum torque ripple on the output. However, the implementation cost is relatively increased because of the reference frame transformation.

On the other hand, the efficiencies of the sensorless schemes could be analytically accessed by using (22), (27) and (31). For a fixed machine, the signal injection frequency could be chosen and fixed based on the rated speed of the machine itself. Then the magnitude of the input voltage could be set in order to collect observable current response to effectively estimate the rotor position. It could be found that the $d$-axis injection has the highest efficiency because it is based on the rotor position error which is a small signal and the reference value is always zero. The rotating injection. method has almost the same magnitude ratio but it is designed based on the sinusoidal position values which will leads large error especially for dynamic operation. Finally, for the stationary axis injection method, it is a simplified 
method but the efficiency depends on the saturation effect or the stator current level and the magnetic material properties of the machine.

In a machine drive system, a compromise is always needed to balance the cost, efficiency and the performance. Fig. 7 shows the overall comparison of the mentioned injection methods, including estimation efficiency (EE), which is compared based on signal/output ratio; implementation cost (IC), which is compared based on the implementation computing cost; signal-process cost (SC), which is compared based on the signal processing unit; torque ripple (TR), which is compared based on the output torque; and independence of machine-structure (IM). A larger vector on each direction indicates higher performance, lower cost, higher efficiency or smaller ripple.

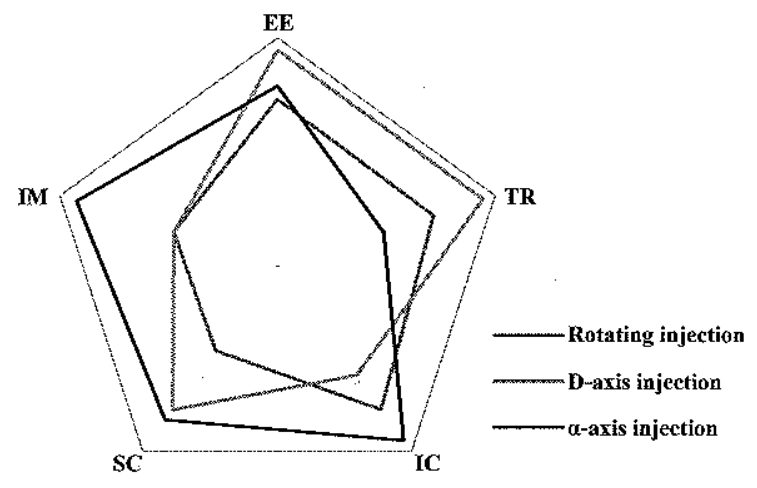

Fig. 7. Overall performance comparison of the injection schemes.

\section{CONCLUSIONS}

This paper aims to develop a comprehensive comparison of three sensorless schemes for PMSM, based on high frequency signal injection. A nonlinear analytical machine model is firstly established and then used as a virtual machine model. High frequency signal injection methods, rotating carrier signal injection method, $d$-axis injection method and the $\alpha$ axis injection method are then applied to the machine and the simulation results are compared.

Not only the estimation efficiency but also the implementation cost, signal process cost and the induced torque ripples are considered. An overall performance graph is developed to demonstrate the results tested based on the comprehensive machine nonlinear model. Both the rotating carrier injection and $d$-axis injection are based on structural saliency and could be applied to IPMSM. The $d$-axis injection method could achieve the best efficiency and minimum torque ripple but the computing cost is the relative large.

On the other hand, the $\alpha$-axis injection method has the easiest implementation process and could be applied to both IPMSM and SPMSM. However the torque ripple is too large. Improved current regulator could be introduced to reduce the ripple.

\section{REFERENCES}

[1] P. Pillay and R. Krishnan, "Control characteristics and speed controller design for a high performance permanent magnet synchronous motor drive," IEEE Trans. Power Electron., vol. 5, no. 2, pp. 151-159, 1990.

[2] C. Lascu and A. M. Trzynadlowski, "Combining the principles of sliding mode, direct torque control, and space-vector modulation in a high-performance sensorless AC drive," IEEE Trans. Ind. Appl, vol. 40 , no. 1 , pp. $170-177$, Jan./Feb. 2004

[3] L. A. Cabrera, M. E. Elbuluk and D. S. Zinger, "Learning techniques to train neural networks as a state selector for inverter-fed induction machines using direct torque control," IEEE Trans. Power Electron., vol. 12 , no. 5 , pp. $788-799$, Sept. 1997 ,

[4] P. B. Schmidt, "Method and apparatus for rotor angle detection," U.S. Patent 6172 498, Jan. 9, 2001

[5] Y. Yan, J. G. Zhu and Y. G. Guo, "A direct torque controlled surface mounted PMSM drive with initial rotor position estimation based on structural and saturation saliencies," in Proceedings of the $41^{s t}$ IEEE IAS Anmual Meeting, New Orleans, LA, Sept. 2007, pp. 683-689.

[6] P. L. Jansen and R. D. Lorenz "Transducerless position and velocity estimation in induction and salient AC machines," IEEE Trans. Ind. Appl., vol. 31, no. 2, pp. 240-247, Mar./Apr. 1995.

[7] Kim Hyunbae, and R. D. Lorenz, 'Carrier signal injection based sensorless control methods for XPM synchronous machine drives,' in Proceedings of the $39^{\text {th }}$ IEEE IAS Annual Meeting, 2004, vol.2, pp. 977-984

[8] J. Ha and S. Sul, "Sensorless field-orientation control of an induction machine by high-frequency signal injection," IEEE Trans. Ind. Appl., vol. 35, no. 1, pp. 45-51, Jan./Feb. 1999.

[9] Ji-Young Lee, Sang-Ho Lee, Geun-Ho Lee, Jung-Pyo Hong, and Jin Hur, "Determination of parameters considering magnetic nonlinearity in an interior permanent magnet synchronous motor," IEEE Trans. Magn, vol. 42, no. 4, pp. 1303-1306, Apr. 2006

[10] Seung-Joo Kim, Hyung-Woo Lee, Kwang-Soo Kim, Jae-Nam Bae, Jong-Bin Im, Cherl-Jin Kim, and Ju Lee, "Torque ripple improvement for interior permanent magnet synchronous motor considering parameters with magnetic saturation," IEEE Trans. Magn., voL. 45, no. 10, pp. 4720-4723, Oct 2009.

[11] A Vagati, M Pastorelli, G. Franceschini, and F. Scapino, "Impact of cross saturation in synchronous reluctance motors of transverselaminated type," IEEE Trans. Ind. Appl.,7 vol. 36, no. 4, pp. 1039 1046, Jul./Aug. 2000

[12] Y. Wang, N. Duan, J. Zhu, Y. Guo, S. Wang, W. Xu, and Y. Li, "Comprehensive Mathematical Model of Permanent Magnet Synchronous Machine Incorporating Structural and Saturation Saliencies," in Proc. $11^{\text {th }}$ Joint MMM-INTERMAG , Washington, DC, USA, Jan, 2010, in press

[13] Y. Yan, J. G. Zhu, Y. Guo and H. Lu, "Modeling and simulation of direct torque controlled PMSM drive system incorporating structural and saturation saliencies," in Proc. IEEE IAS Annu Meeting, Tampa, FL, Oct 2006, pp. 76-83. 